\title{
A Comparative Review on Routing Protocols in MANET
}

\author{
Rajeshwar Sharma \\ Assistant Professor \\ PG department of computer \\ science, \\ DAV College Jalandhar, India
}

\author{
Tarun Sharma \\ Department of Computer \\ Science \& Engineering, \\ CT Institute of Technology \& \\ Research, \\ Jalandhar, India
}

\author{
Aditi Kalia \\ Assistant Professor, \\ Department of Computer \\ SCIENCE \& Enginering, \\ D.Y patil college of \\ Engineering, \\ Pune, India
}

\begin{abstract}
Routing in MANETs is a one of the dynamically and demanding task and has received a great amount of awareness from researchers around the globe. To overcome this problem, a various number of routing classes have been introduced and the number is still rising day by day fastly. It is quite hard to decide which protocols or routing classes may do well under an amount of diverse network scenarios such as network volume and network topology etc. In this paper, we present a summary of a large range of the existing routing classes with a particular focus on their uniqueness and their functionality. Also, the judgment is provided based on the routing functionality and information is used to build routing decisions. The presentation of all the routing protocols or classes is also discussed. Further this study will assist the researchers to get a summary of the existing classes and advice which protocols may execute better with respect to varying between network scenarios.
\end{abstract}

\section{General Terms}

MANET , Routing protocols, comparison of Routing protocols

\section{Keywords}

Mobile ad-hoc Network, Routing protocol, classification of protocol.

\section{INTRODUCTION}

MANETs is one of the most prominent fields for research in modern era and growth of the wireless network. As the popularity of the mobile ad-hoc network (MANET) will be increasing day by day. Now it becomes one of the most spirited and sporty fields of communication in the wireless network. MANET is self-arrange and decentralized networks or infrastructure less network. There are no conditions on these nodes to unite or leave the network. Nodes are free to move independently and change its links with other devices or nodes frequently. MANET provides a routable process for exchange of packets from one node to another, because of there is wireless networking environment [1]. Mobile Ad-Hoc networks are extremely dynamic networks characterized by the nonappearance of physical infrastructure. The functionality of Mobile Ad-Hoc Network finds the routes and maintains the routes with the help of routers. in such networks, nodes are capable of moving and coordinating with their neighbors [2]. MANET each node activate both as host as well as a router to forward packet to intermediate nodes, with the uniqueness of self-configuring and self-club which enable it to form a new network speedily. Speedy and easy establishment of such networks makes them possible to use in military, disaster area recovery and in another environment where no physical infrastructure exists [7].

Applications of MANETS diverge from commercial use, private sector to military and emergency purposes. [3]. with MANET, Such as Business application, military application, emergency operations, home, office, educational application and wireless sensor networks etc [1]. The key issues related to Ad-hoc routing protocols are routing technique to be used that is unicast routing or multicast routing, dynamic network topology which changes when mobile nodes move from one BSS to other BSS as well as speed of mobile nodes. The quality of Service (QoS) is also the important MANET performance parameter for controlled traffic flow. Other than that some other challenges related to MANETs are Frequency of updates or Network overhead, Scalability, Mobile agent based routing, Energy efficient/Power-aware routing, Secure routing [4].

This Paper is structured as follows; section 1 Introduction of MANET, Section II Discuss Some Characteristics of MANET, Section III Related Work, Section IV Comparison of different Routing protocols in MANET, Section V Conclusion.

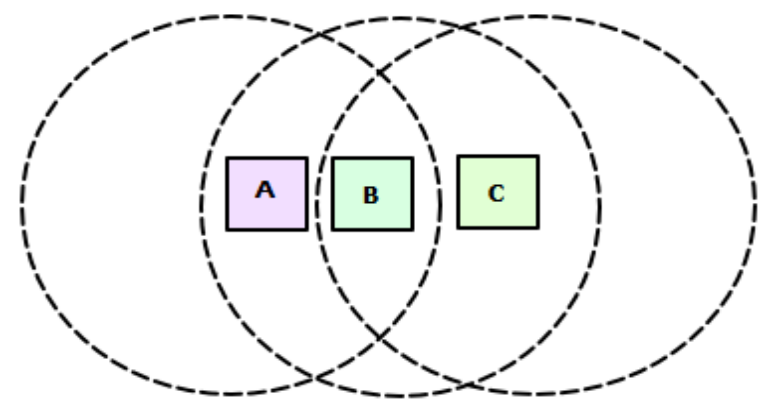

Figure 1: A Mobile Ad-hoc Network (MANET)

\section{CHARACTERISTICS OF MANETs}

The design of routing protocol for MANET is tricky because of numerous network limitations. MANET experience from the restrictions of several network resources, for examples, energy, bandwidth, processor, and storage. The aim of challenges in sensor networks involves the following main aspects .

\subsection{Dynamic topology}

Nodes are free to move arbitrarily in any direction thus the topology of the network change unpredictably.

\subsection{Limited Bandwidth}

The bandwidth available for wireless networks is generally low than that of wired networks. The throughput of these networks is generally low due various noises, fading effects.

\subsection{Energy constrained operation}

The nodes are portable devices and are dependent on batteries. This is the most important design consideration of the MANET 


\subsection{Security}

wireless networks are more prone to threats Than wired networks. The increased possibility of Various security attacks like eavesdropping, denial of

Service should be handled carefully. The performance of MANET depends on the routing protocol, battery consumption by the nodes. There are Various Quality of service parameters which affect the performance like a bandwidth delay, jitter, throughput etc. Due to dynamic topology routing is the major challenging these networks because the bandwidth provided to the nodes at one point of time becomes unavailable if the nodes move from a particular position and go to other position. Moreover, routing affects the performance of these networks. Therefore efficient routing protocol needs to be developed to meet all these challenges. routing protocol in MANET is classified into three categories on the basis of route discovery reactive also called as the on-demand routing protocol ,proactive also known as the table driven protocol and Hybrid protocol. Further classification of routing protocols is done on the basis of network organization as flat based, hierarchical based and location based. In flat based protocol all the nodes are equal i.e. they play the same role in the network. In hierarchical protocol different nodes play different roles i.e. in this different cluster heads are chosen among cluster members. In location-based protocol nodes rely on the location information and use this information for communication.

\section{LITRATURE REVIEW}

The different pieces of key literature in the field of MANET routing protocols has been examined in the current idea within the field. Maghsoudlou A. et al. [2001] surveyed on the unlike face routing algorithms as well as dissimilar face routing strategies and greedy routing algorithms under geographical routing protocol in MANET. Authors experiential the geographic routing protocols are based on the greedy forwarding in which the data is sent to the nearest node of the target, but sometimes the data could be tarnished. if there is no foreigner node near to the target. Authors also proposed to improve strategy to recuperate from this state and concluded that the most common strategy to recover from the state of the void is faced routing algorithm which uses the planner graphs [6].

Chaudhary P. et al; [2014] performed the assessment of AODV, DSR (reactive) and DSDV (proactive) routing protocols. These are based on Packet Delivery Ratio, standard end to end delay under the different mobility model with varying the speed of mobile. These routing protocols are measurable efficiently. Simulation is done using network simulator-2(NS-2). AODV performs better as compared to DSR and DSDV in mobility model. The packet delivery ratio is0 high of AODV in random walk and random direction. But the end to end delay was also very high for AODV protocol. So the overall performance of DSR is better than the AODV and DSDV in random walk and random direction mobility model [7].

Khan J. et al. [2011] In this paper we not only evaluate the performance of ad-hoc routing protocols class in order to establish its accuracy, effectiveness, traffic load and the end to end delay in energetic intermediate nodes scenario but also to apply OPNET simulator in AODV and DSR routing classes. Opnet simulator is proposed by author to observe performance with respect to different parameters that changes mobility models have important impact on their performance of both AODV and DSR routing class could be the most excellent solution in MANET, instead of separate presentation of both
AODV and DSR routing class and also in intermediate nodes data transport rate from source to target [13].

Mittal P. et al. [2013] presented the comparison of MANET routing protocols i.e. GRP, AODV, OLSR and DSR on the basis of end-to-end delay, network load, retransmission attempts, and throughput by using simulation tool OPNET modeler 14.5. Authors accomplished that AODV and DSR perform better than as compared to other protocols. The throughput of AODV and DSR is more than as compared to other protocols and delay of AODV is minor than as that of other protocols [8].

Menon V. G. et al. [2013] analyzed the performance of the different geographic routing protocols in high mobility. Authors had been compared the performance of different geographic routing protocols on the basis of performance metrics and listed the merits and demerits of these protocols on the basis of their performance metrics. Authors had been discussed the different parameters involved for scheming and choosing a routing protocol [3].

Wadhwa D. et al. [2014] compared different geographic routing protocol such as Location-aided routing, Greedy perimeter stateless routing, and Energy-aware geographic routing on the basis of performance metrics such as system lifetime, the end to end delay and packet delivery ratio and energy utilization by using simulation tool NS2. Authors concluded that the geographic routing gives high packet delivery ratio, better energy utilization and better network lifetime as compared to other protocols when the topology changes dynamically and when the mobility is high [15].

\section{ROUTING PROTOCOLS FOR MANETS}

Routing is an action to move the information from source point to destination point within the network. During this process, at least, one intermediate node within the network is encountered. The routing notion basically involves, two activities: firstly, determining best possible routing paths and secondly, transferring the information throughout a network. Routing basically divided into two types: static routing and dynamic routing. Static routing refers to the routing policy being manual. In static routing maintains a routing table by the administrator. Dynamic routing mainly depends upon the state. Mobile ad hoc network present the dynamic routing [10]. These protocols can be divided into three classes' proactive class, reactive class and hybrid class as shown in figure 2.This classification of routing protocols are work according to their technique such as hop count, link state and QoS in route discovery. Hop count method, each node contains next hop information in its routing table, to the destination. While link state routing protocols keep a routing table for absolute topology, which is built up by finding shortest path of link costs. QoS routing is the procedure of selecting the path to be used by the packets of a flow, based on its QoS requirements eg bandwidth, delay etc [4].

\subsection{Proactive Routing Protocol}

In the table-driven routing protocols, each ad hoc node Consists of a topology table, which contains the up to date networks nodes communication information. This table is updated all the time and it gives the proactive protocols another name of table-driven. One or more routing tables are maintained at each node and are exchanged frequently to share the topology information with the neighboring nodes in organize to maintain a consistent network view $[4,9]$. If a route 
has already existed before traffic arrives, a transmission will occur without delay. Otherwise, traffic packets should stay in queue until the node receives routing information corresponding to its destination. The various proactive routing protocols are Destination-Sequenced Distance Vector (DSDV), Wireless Routing Protocol (WRP), Optimized link state routing (OLSR) [9].

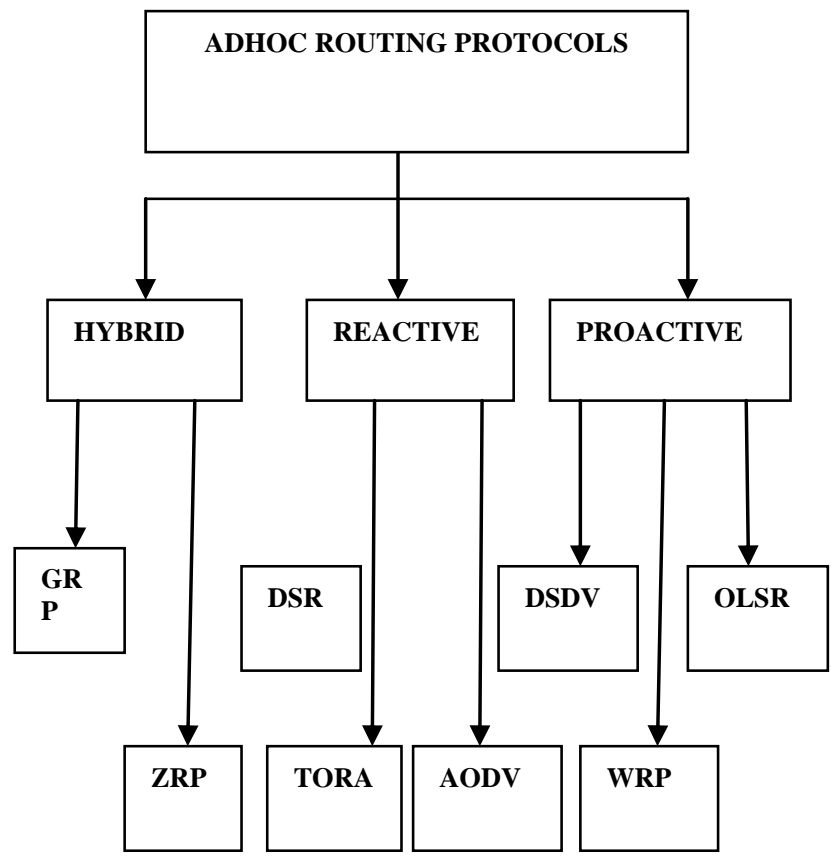

Figure 1: Routing Algorithm in MANET

\subsubsection{DSDV}

DSDV is Proactive routing class and this protocol is planned by Perkins and Bhagwat. The Destination-Sequenced DistanceVector (DSDV) routing class [14].Routing protocol class is based on the proposal of the classical Bellman-Ford routing Algorithm with definite improvements such as formation it loop- free. The distance vector routing class is less robust as comparatively link state routing class due to troubles such as count to infinity and bouncing outcome. In this, each system maintains a routing related table containing entries for all the policy in the network. In organize to keep the routing table entirely reorganized at all the time each device occasionally broadcasts different routing communication to its neighbor devices. When a neighbor device receives the broadcasted different routing message from the sender and knows the existing relation cost to the device, it compares this value and the related value stored in its routing table.

\subsubsection{OLSR}

OLSR is the proactive class that employs a capable link state packet forwarding system called multipoint relaying [11, 12]. It optimizes the clean link state routing class. There are two ways to done Optimizations: by reducing the volume of the control packets and the Second way by tumbling the number of associations used for promoting the link state packets. As you know that each and every node preserves the topology information about the network by periodically replacing linkstate communication among the other nodes. OLSR routing class is based on the following three mechanisms: neighbor sensing, capable flooding, and calculation of an optimum route using the variety of shortest-path algorithm. Neighbor sensing is the judgment of changes in the region of the node. Each node finds out the best route to every known target using this topology information and accumulates this information in a routing table. The shortest path algorithm is then used for calculating the most constructive path. Routes to every destination are directly available when data broadcast begins furthermore remaining suitable for a specific period of time till the information is ended.

\subsubsection{WRP}

The Wireless Routing Protocol class, as planned by GarciaLuna-Aceves [17], it is a table-based class related to DSDV class that take over the assets of Bellman- Ford Algorithm. The main aim is preserving routing information among a variety of nodes in the network about the shortest path to every target. Wireless routing protocols (WRP) is a loop-free routing class. WRP is a path-searching algorithm with the exemption of shunning the count-to-infinity disaster by forcing each node to execute constancy checks of precursor information detailed by all its neighbors. Each and every node in the network implies a set of four tables to carry on more precise information. these are following tables Distance table (DT), Routing table (RT), Link-cost table (LCT), Message retransmission list (MRL) table. In that case of link breakdown between two nodes, the nodes launch bring up to date communications to their neighbors. WRP fit into the class of path searching algorithms with a significant exception. It counters the count-to-infinity difficulty by forcing each node to do consistency checks of precursor information statemented by all its neighbors. This removed looping circumstances and enables quicker route convergence when a link failure occurs.

\subsection{Reactive Protocols}

On-demand routing these protocols do not replace routing information at times. but use flooding method to obtain information when mandatory for a node to send the data packet. The host node which needs to broadcast packets to a destination in the network broadcasts a route request to all nodes in the network. The host node will be waiting for the reply of the nodes in the network to provide a path to target before transmitting packets [13]. They create a route during a route discovery procedure. In which route request packets are flooded throughout the network starting with the immediate neighbors of the source. Once a route is formed or multiple routes are found for the destination, the route discovery process comes to an end. A route maintenance process maintains the stability of the route for the time span it is needed from the source. Some of the examples of the source- initiated routing protocols are DSR, AODV and TORA etc [16].

\subsubsection{AODV}

AODV is important routing class in Reactive protocol and it builds routes via a route request/ route reply query sequence. When a source node wants a route to a target for which it does not already have a path, it transmits a route request (RREQ) packet to the network. Nodes getting this packet bring up to date their information for the source node and set up backward pointers to the initial node in the route tables. In calculation to the source node's IP address, current series number, and broadcast ID, the RREQ also includes the most recent series number for the target of which the source node is alert. A node getting the RREQ may launch a route reply (RREP) if it is either the target or if it has a route to the destination with the equivalent series number greater than or equal to that restricted in the RREQ. If this is the case, it unicasts an RREP back to the source node, otherwise, it retransmits the RREQ. Nodes keep the path of the RREQ's source node IP address and transmit ID of a better route. If they get an RREQ which they have already progressed, they reject the RREQ and don't advance it. As long as the route runs lively, it will continue to be preserved. A 
route is calculated energetic as long as there are data packets at times traveling from the source to target along that way. Once the source node is stopped transfer data packets, the links will time out and finally be deleted from intermediate node routing tables. After receiving the RERR, if source node still desires route, it can reinitiate route discovery $[18,19]$.

\subsubsection{TORA}

The Temporally-Ordered Routing Algorithm (TORA) routing class. It was developed by Park and Corson. Temporarily ordered routing algorithm (TORA) is the class that truly adaptive, loop-free, discrete routing algorithm based on the concept of connection turnaround. It uses directed acyclic graphs (DAG) to explain the routes either upstream as well as downstream. However to give this feature, TORA wants synchronization of the nodes which restrictions the function of the protocol. TORA routing class is a moderately complex protocol but what makes it unique and important is its main characteristic of the broadcast of manage messages only around the point of the crash when a link stoppage occurs. In the evaluation, all the other protocols require to re-initiate a route detection when a link not succeed but TORA would be able to patch itself up around the point of failure. This characteristic allows TORA to level up to bigger networks, but it has a higher overhead for smaller networks. TORA occupy four key operations: creating, preserving, removing and optimizing routes. Since every node must have a height, any node which does not have a height is calculated as a removed node and its height is considered as null. Sometimes the nodes are given new heights to get better the linking structure. This purpose is called optimization of routes.

\subsubsection{DSR}

DSR is one of the purest examples of an on-demand routing protocol that is based on the concept of source

routing. It is designed especially for use in multihop ad hoc networks of mobile nodes. It allows the network to be completely self-organizing and self-configuring and does not need any existing network infrastructure or administration. DSR uses no periodic routing messages like AODV, thereby reduces network bandwidth overhead, conserves battery power and avoids large routing updates. Instead, DSR needs support from the MAC layer to identify link failure. DSR is composed of the two mechanisms of Route Discovery and Route Maintenance, which work together to allow nodes to discover and maintain source routes to arbitrary destinations in the network. DSR has a unique advantage by virtue of source routing. As the route is part of the packet itself, routing loops, either short - lived or long - lived, cannot be formed as they can be immediately detected and eliminated. This property opens up the protocol to a variety of useful optimizations. Neither AODV nor DSR guarantees the shortest path. If the destination alone can respond to route requests and the source node is always the initiator of the route request, the initial route may the shortest [21].

\subsection{Hybrid routing protocol}

There are two classes in the hybrid routing protocol. First one is proactive and second one is reactive class Hybrid routing protocols are the combination of proactive protocols and reactive protocols. Hybrid routing protocol has benefited both proactive and reactive routing protocols. Firstly it performs like proactive routing protocol because in initial nodes have tables. Then whenever nodes find that they do not have routes to target, they start route discovery and behave like reactive routing protocols. The hybrid protocol is ZRP [7]. Which brings the benefit of both the approaches together is ZoneBased Hierarchical Link-State Routing Protocol (ZRP). ZRP defines each node a zone around itself containing all neighbor nodes with certain ' $\mathrm{k}$ ' hop $(\mathrm{k}=1,2$ or 3$)$. If the destination node's location is within the zone of the source then it uses proactive routing else it uses reactive routing protocol [1].

\subsubsection{ZRP}

Haas and Pearlman projected Zone Routing class. ZRP [22] It is a hybrid routing class for mobile ad hoc Networks which restricts the nodes into sub-networks (zones). It includes the qualities of on-demand and proactive routing class. Within every zone, proactive Networks which restricts the nodes into sub-networks (zones). It incorporates the qualities of ondemand and proactive routing protocols. Within each zone, proactive routing class is modified to speed up communication surrounded by neighbors. The inter-zone contact uses ondemand routing classes to reduce unnecessary communication. The network is separated into routing zones according to detachment between mobile nodes. specified a hop distance $d$ and a node $\mathrm{N}$, all nodes within communication hop distance at most $\mathrm{d}$ from $\mathrm{N}$ fit into the routing zone of $\mathrm{N}$. Peripheral nodes of $\mathrm{N}$ are N's neighboring nodes in its routing zone which are accurately $\mathrm{d}$ hops away from $\mathrm{N}$. a significant issue of zone routing is to resolve the size of the zone. A better zone routing protocol, Independent Zone Routing (IZR), which permits adaptive and stretch reconfiguration of the reduced size of the zone, is introduced in [23]. Besides, the adaptive nature of the IZR class enhances the scalability of the ad hoc network. Each node rarely wants to update the routing information surrounded by the zone. Additionally, some limited route optimization is performed at each node, which includes the following actions: removal of unneeded routes, shortening of routes, spotted of link failures.

\section{CONCLUSION AND FUTURE SCOPE}

This article described the classification of several routing schemes according to the routing strategy. We discussed some important characteristics of the three routing strategies such as Reactive, proactive and Hybrid protocols Table 1 highlighted few differences between them In this paper, an effort has been made to concentrate on the comparative study of DSDV, AODV, DSR, TORA, OLSR, WRP, DSDV. Moreover, a single routing protocol can't perform best in all situations. So, the choice of routing protocol should be done carefully according to the requirements of the specific application The focus of the study in our future research work is to propose an extension of the existing conventional routing protocols which will be better in terms of security, throughput, efficient utilization of limited resources and quality of service 
Table 1: Comparison of Routing Protocols in MANETS

\begin{tabular}{|l|l|l|l|l|l|l|}
\hline parameter & DSDV & WRP & OLSR & AODV & DSR & TORA \\
\hline $\begin{array}{l}\text { Routing } \\
\text { structure }\end{array}$ & Flat & Flat & Flat & Flat & Flat & Flat \\
\hline $\begin{array}{l}\text { Routing } \\
\text { overhead }\end{array}$ & high & high & Low & high & high & High \\
\hline $\begin{array}{l}\text { Caching } \\
\text { overhead }\end{array}$ & Medium & high & High & Low & high & High \\
\hline $\begin{array}{l}\text { Throughput } \\
\text { Low }\end{array}$ & Low & High & High & Low & Low \\
\hline Loop Free & Yes & Yes & Low & Yes & Yes & Yes \\
\hline
\end{tabular}

\section{REFERENCES}

[1] Sumathia K. and Priyadharshinib A.," energyoptimization in manets using ondemand routingprotocol" elsevier, procedia computer science 47 (2015 ) $460-470$.

[2] dhawan s. and saroha v.," optimize the routingprotocol (grp, olsr, dsr) using opnet \& itsperformance evaluation", international journal ofadvances in engineering \& technology, vol. 6, issue3, pp. 1399-1408.

[3] Menon V. and Prathap J., "Performance Analysis ofGeographic Routing Protocols in Highly Mobile AdHoc Network", Journal of Theoretical and AppliedInformation Technology, Vol. 54. No.1,10August,2013

[4] Al-Khwildi A. and Al-Raweshidy H., "An EfficientRouting Protocol for Wireless Ad hoc Networks",4th International Conference: Sciences ofElectronic, Technologies of Information andTelecommunications March 25-29, 2007 - tunisia

[5] Singh G. and Singh J.“ MANET: Issues andBehavior Analysis of Routing Protocols",International Journal of Advanced Research inComputer Science and Software Engineering,Vol. 2, Issue 4, April 2012.

[6] Khatkar A andSingh Y., "Performance Evaluationof Hybrid Routing Protocols in Mobile AdhocNetworks",Advanced Comput. CommunicationTechnologies(ACCT- 2012), DOI $10.1109,2012$.

[7] Chudhary P. and Singh V., “ Performance Evaluationof Mobile Ad- hoc Networks Routing ProtocolAODV, DSR \& DSDV in Different MobilityModels with Varying Speed of Nodes.",International Journal of Computer Science andInformation Technologies,Vol. 5 (3) , 2014, 3351-3354.

[8] Mittal P.,Singh P. and Rani S., "PerformanceAnalysis of AODV, OLSR, GRP and DSR RoutingProtocols With Database Load in MANET"International Journal of Research in Engineeringand Technology(IJRET), eISSN: 2319-1163,Vol. 02 Issue: 09 Sep, 2013.

[9] A. K. Gupta, H. Sadawarti, and A. K. Verma,"Performance analysis of AODV, DSR \&
TORARouting

Protocols,"

"IACSIT InternationalJournal of Engineering and Technology", vol.2,April 2010.

[10] Kaur S., Bhatia N. and Kapoor N.“ SimulationAnalysis of AODV Routing Protocol of MANETusing OPNET”, IJCST Vol. 2, Issue 3, September2011

[11] L. Abusalah, A. Khokhar and M. Guizani, "ASurvey of Secure Mobile Ad Hoc RoutingProtocols", "IEEE Communications Surveys\& Tutorials", vol. 10 no. 4, 4th Quarter 2008.

[12] T. Clausen, P. Jacquet, A. Laouiti, P. Muhlethaler,A. Qayyum, L.Viennot, "Optimized link staterouting protocol for ad hoc networks," in:"Proceedings of IEEE INMIC", December 2001,pp. 62-68.

[13] khan $\uparrow$ J., Hyder $\uparrow \uparrow$ S. and Mustafa $\uparrow$ S., “ Modelingand Simulation Of Dynamic Intermediate NodesAnd Performance Analysis in MANETS ReactiveRouting protocols", International Journal of Gridand Distributed Computing., Vol. 4, No. 1, March2011.

[14] S. Murthy, C. Siva Ram and B.S. Manoj, "Ad HocWireless Networks: Architectures and Protocols,"Prentice Hall, Chapter 7, 2004.

[15] Wadhwa D., Deepika, Kochher V.and Tyagi R., "AReview of Comparison of Geographic RoutingProtocols in Mobile Ad-hoc Network", Advance inElectronic and Electric Engineering, ISSN 2231-1297, Vol.4, pp. 51-58, 2014.

[16] Gupta A. and Sharma S.,"A Survey on LocationBased Routing Protocols in Mobile Ad-hocNetworks International Journal of ComputerScience and Information Technologies, Vol. 5 (2) ,2014, 994-997.

[17] S. Murthy and J. J. Garcia-Luna-Aceves, "An Efficient Routing Protocol forWireless Networks," "ACM Mobile Networksand App.J., Special Issue on Routing in MobileCommunication Networks", Oct. 1996, pp. 18397.

[18] Perkins C., Royer E. B. and Das S., "Ad hoc On-Demand Distance Vector (AODV) Routing -Internet Draft," IETF Network Working Group,RFC 3561, pp.1-38, 2003. 
International Journal of Computer Applications (0975 - 8887)

Volume 133 - No.1, January 2016

[19] Abolhasan, M., Wysocki, T., Dutkiewic, E. (2004),"A Review of Routing Protocols for Mobile Ad - Hoc Networks,"ELSEVIER,Ad-hoc Networks(2004), vol. 2, pp. 1-24.

[20] A. K. Gupta, H. Sadawarti, and A. K. Verma,"Review of Various Routing Protocols forMANETs," "International Journal ofInformation and Electronics Engineering, Vol. 1,No. 3, November 2011

[21] M.Khiavi,S.jamali,S.jahansakhsh,Gudahakriz,"Performa ce comaprision of AODV,DSDV,DSRand TORA routing protocols in MANETs.",Int.Res Jr. of Appl and basic sciences, Vol 3, pp.1429-1436,2012

[22] Z. J. Haas and M. R. Pearlman, .ZRP: a hybridframework for routing in ad hoc networks, pp.221.253, 2001.

[23] P. Samar, M. R. Pearlman, and Z. J. Haas,"Independent zone routing: an adaptive hybridrouting framework for ad hoc wireless networks," "in IEEE/ACM Transactions on Networking (TON)", vol. 12, 2004, pp. 595.608. 